\section{Mortalidade infantil em uma amostra de nascimentos do município do Rio de Janeiro, 1999-2001: "linkage" com o Sistema de Informação de Mortalidade}

\section{Infant mortality in a sample of births in the municipality of Rio de Janeiro 1999-2001: linkage with the Mortality Information System}

Ana Paula Esteves Pereira1

Silvana Granado Nogueira da Gama²

Maria do Carmo Leal3

1 Vice-presidência de ensino, informação e comunicação Fundação Oswaldo Cruz, FIOCRUZ. Rio de Janeiro, RJ, Brasil. 2,3 Departamento de Epidemiologia. Escola Nacional de Saúde Pública. Fundação Oswaldo Cruz, FIOCRUZ. Rua Leopoldo Bulhões, $1480.8^{\circ}$ andar. Rio de Janeiro, RJ, Brasil. CEP: 21.041-210 E-mail: granado@ fiocruz.br

\begin{abstract}
Objectives: to estimate neonatal and postneonatal mortality rates (NMR and PNMR) of a sample of births in the municipality of Rio de Janeiro, 1999-2001, and to compare them according to the type of maternity and access to hospital delivery and to describe deaths according to their primary cause.

Methods: the identification of infant deaths in the Mortality Information System (MIS) was accomplished through a probabilistic record linkage method. Bivariate analysis was applied with calculation of relative risks $(R R)$ and respective $95 \%$ confidence intervals.

Results: neonatal and post-natal mortality rates were seven and six times higher in public than in private maternities respectively. For puerperal women non-resident in the municipality and those who were denied hospital admittance in other maternity hospitals, IMR was twice as high. High IMR caused by affections of the perinatal period (PPA) even in children weighing over 1500 grams (2.3 of 1.000 born alive infants). Neonatal deaths by PPA were six times more frequent in public maternity hospitals than in private ones.

Conclusions: the enormous differences in neonatal and post-neonatal mortality rates between public and private hospitals point towards the need for reduction of social inequalities and improvement of the access to healthcare as fundamental issues to be approached in the municipality of Rio de Janeiro.
\end{abstract}

Key words Neonatal mortality, Post-Neonatal mortality, Information systems, Linkage

\section{Resumo}

Objetivos: estimar as taxas de mortalidade neonatal (TMN) e pós-neonatal (TMPN) em uma amostra de nascimentos do município do Rio de Janeiro, durante o perído de 1999-2001, compará-las segundo o tipo de maternidade e acesso ao parto hospitalar e descrever os óbitos segundo a sua causa básica.

Métodos: a identificação dos óbitos infantis no Sistema de Informação sobre Mortalidade (SIM) foi realizada por meio do método de relacionamento probabilístico de registros. A análise foi bivariada, sendo calculados os riscos relativos $(R R)$ e seus respectivos intervalos de confiança $95 \%$.

Resultados: as taxas de mortalidade neonatal e pósneonatal foram sete e seis vezes maiores nas maternidades públicas que nas privadas, respectivamente. Para as puérperas não residentes no município e aquelas que tentaram "parir" em outros estabelecimentos, sem sucesso, a TMN foi duas vezes maior. Observou-se elevada TMN por afecções do período perinatal (APP), mesmo em crianças com mais de $1500 \mathrm{~g}$ (2,3 por mil $N V$ ). Óbitos neonatais por APP foram seis vezes mais freqüentes nos hospitais públicos que nos privados.

Conclusões: a enorme diferença nas taxas de mortalidade neonatal e pós-neonatal entre os hospitais públicos e privados aponta, como fatores primordiais a sofrerem intervenção para a retomada da redução dessas taxas no município do Rio de Janeiro, a redução das desigualdades sociais e de acesso.

Palavras-chave Mortalidade neonatal, Mortalidade pós-neonatal, Sistemas de informação, Linkage 


\section{Introdução}

A taxa de mortalidade infantil (TMI) no município do Rio de Janeiro não decaiu no período de 2001 a 2004, a qual se manteve em torno de 15 óbitos por mil nascidos vivos (NV). ${ }^{1}$ Esse valor ainda é alto quando comparado a municípios nos quais o patamar de menos de 11 óbitos por mil NV já foi atingido, como Curitiba (11 por mil NV) e Florianópolis (oito por mil NV). ${ }^{1}$ É urgente reduzir essa taxa, já que muitas dessas mortes são potencialmente evitáveis, ora decorrentes de causas perinatais $(68 \%)$ ora de doenças infecto-parasitárias e respiratórias (8\%) (dados de 2004). ${ }^{1}$ Assim, identificar grupos mais expostos aos diversos fatores de risco e características que determinam as diferentes causas de óbito é imprescindível.

O relacionamento de dados tem se mostrado um importante instrumento metodológico em estudos com enfoque na saúde infantil, unificando diferentes fontes de informações como o Sistema de Informação de Mortalidade (SIM) e o Sistema de Informação de Nascidos Vivos (SINASC). ${ }^{2}$ A utilização de dados primários é oportuna, já que o banco de dados gerado pelo SINASC ainda possui algumas limitações, além da qualidade do seu preenchimento não ter atingido um nível satisfatório para algumas variáveis. 3

Este trabalho tem como objetivo estimar a taxa de mortalidade neonatal e pós-neonatal na população do "Estudo da Morbi-mortalidade e da Atenção Peri e Neonatal no Município do Rio de Janeiro",4 compará-las segundo algumas variáveis de acesso e tipo de serviço, além de descrever a causa básica dos óbitos segundo o muito baixo peso ao nascer e o perfil da maternidade.

\section{Métodos}

O "Estudo da Morbi-mortalidade e da Atenção Peri e Neonatal no Município do Rio de Janeiro", foi desenvolvido com base em uma amostra de puérperas que se hospitalizaram em maternidades do município do Rio de Janeiro, RJ, Brasil, por ocasião do parto, entre julho de 1999 e março de 2001. Os dados foram coletados dos prontuários maternos e dos recém-nascidos, assim como a partir de entrevistas com as mães, no pós-parto imediato.

A amostragem foi estratificada, agrupando-se os estabelecimentos de saúde em três perfis de maternidades: as municipais e federais; as estaduais, militares, filantrópicas e privadas conveniadas com o Sistema Único de Saúde (SUS) e, por último, as maternidades privadas não conveniadas com o SUS. Em cada estrato foi selecionada uma amostra de, aproximadamente, $10,0 \%$ de parturientes do número previsto de partos em todos os hospitais de cada estrato. Foram excluídos da amostra aqueles estabelecimentos/hospitais com menos de duzentos partos por ano, correspondente a apenas 3,7\% do total.

Ao final da pesquisa foram realizadas 10.072 entrevistas. As perdas contabilizaram $4,5 \%$ do total de partos ocorridos. Ao todo foram selecionadas 47 instituições: 12 compuseram o primeiro estrato amostral, correspondendo a 34,8\% dos partos; $10 \mathrm{o}$ segundo estrato, correspondendo a $34,4 \%$ dos partos; e 25 o terceiro, com $30,8 \%$ dos partos.

Excluída a parcela da amostra cujo desfecho correspondeu ao óbito fetal, 9.969 puérperas compuseram a análise deste artigo. Considerando os partos múltiplos, o total de nascidos vivos correspondeu a 10.186 .

Para a inclusão do desfecho "óbito infantil", foi realizado um linkage entre dois bancos de dados: $10.186 \mathrm{NV}$ dessa amostra e 5946 óbitos infantis ocorridos no município do Rio de Janeiro no período de junho de 1999 a março de 2002. Os dados sobre mortalidade infantil foram coletados a partir do Sistema de Informações de Mortalidade, fornecido pela Coordenação de Epidemiologia da Secretaria Municipal de Saúde do Rio de Janeiro.

O linkage dos dados foi feito pelo método do relacionamento probabilístico de registros, utilizando o conceito de escore limiar proposto por Newcombe et al. 5 Os registros foram trabalhados segundo os passos descritos por Camargo e Coeli. 6 Utilizou-se para tal o programa Reclink II. 6

Para o cálculo da taxa de mortalidade neonatal utilizou-se como numerador os óbitos ocorridos do nascimento até o $27^{\circ}$ dia; para a pós-neonatal, os ocorridos do $28^{\circ}$ até o $12^{\circ}$ mês incompleto. Para os dois componentes foi utilizado como denominador o total de nascidos vivos da amostra.

Para cada categoria das variáveis obteve-se a taxa de mortalidade neonatal e pós-neonatal. A análise foi bivariada, sendo calculados os riscos relativos (RR) e seus respectivos intervalos de $95 \%$ de confiança.

Este estudo foi submetido ao Comitê de Ética para Pesquisa da Escola Nacional de Saúde Pública, Fundação Oswaldo Cruz. A confidencialidade da informação quanto ao conteúdo e à identificação da puérpera, assim como o anonimato por ocasião da publicação dos resultados, foi assegurado. 


\section{Resultados}

Oitenta óbitos neonatais e 14 pós-neonatais foram identificados no trabalho de campo. No processo de "linkage" foram resgatados 28 óbitos neonatais e 42 óbitos pós-neonatais, totalizando 164 óbitos infantis.

A identificação desses óbitos ampliou em $20 \%$ a taxa de mortalidade neonatal precoce estimada no trabalho de campo, que passou de 5,7 para 6,8 óbitos por mil NV, e também viabilizou a estimativa das taxas de mortalidade neonatal (10,6 por mil NV) e infantil (16,1 por mil NV).

Na amostra estudada a TMI (16,1 óbitos por mil NV), e também a taxa de mortalidade pós-neonatal $(5,5$ por mil NV), se assemelharam à taxa encontrada no município do Rio de Janeiro no ano de 2000, que foi de 16,7 e 5,5 óbitos por mil NV, respectivamente1. Já para o componente neonatal, a taxa na amostra (10,6 por mil NV) foi um pouco menor do que aquela encontrada no município, de 11,2 óbitos por mil NV.1

Dentre os 94 óbitos identificados no trabalho de campo, oito $(8,5 \%)$ não tiveram suas declarações de óbito encontradas no SIM. Outra discrepância encontrada refere-se a quatro (5\%) dos 80 óbitos classificados como neonatais no trabalho de campo, e que foram notificados como fetais no SIM.

Considerando o perfil das maternidades, as taxas de mortalidade neonatal e pós-neonatal foram sete e seis vezes maiores nas municipais e federais que nas privadas não conveniadas ao SUS, respectivamente (Tabela1).

Nas maternidades que dispunham de UTI neonatal a taxa de mortalidade neonatal foi $75 \%$ maior do que naquelas sem esse recurso. Já para as puérperas oriundas de outros municípios que não o Rio de Janeiro essa taxa foi 2,2 vezes maior do que entre as residentes neste município (Tabela1).

Entre os filhos daquelas que peregrinaram (tentaram realizar o parto em outros estabelecimentos sem sucesso) a taxa de mortalidade neonatal foi 2,4 vezes maior do que nas que não o fizeram.

$\mathrm{Na}$ análise da mortalidade infantil por sua causa básica, observou-se um alto percentual de óbitos com classificação mal definida (6\%), sendo esse ainda maior para o componente pós-neonatal (18\%) (resultado não apresentado em tabela).

Dentre os óbitos com a causa básica definida, as afecções do período perinatal (APP) foram a principal causa para o componente neonatal $(77,8 \%)$, seguida pelas mal-formações congênitas $(18,2 \%)$. Para o pós-neonatal as APP perdem importância,

\section{Tabela 1}

Mortalidade infantil segundo o tipo e complexidade do prestador, local de residência materna e peregrinação para o parto.

\begin{tabular}{|c|c|c|c|c|c|c|c|}
\hline & \multicolumn{3}{|c|}{ Neonatal } & \multicolumn{3}{|c|}{ Pós-neonatal } & \multirow[b]{2}{*}{ Nascidos Vivos } \\
\hline & Taxa & $\mathrm{RR}$ & IC95\% & Taxa & $\mathrm{RR}$ & IC95\% & \\
\hline \multicolumn{8}{|c|}{ Tipo de prestador } \\
\hline Estrato 3 & 2,8 & 1 & - & 1,6 & 1 & - & 3162 \\
\hline Estrato 2 & 7,9 & $2,78 *$ & $1,54-5,03$ & 4,7 & $2,98 *$ & $1,24-7,19$ & 3412 \\
\hline Estrato 1 & 19,9 & 7,00 * & $4,48-10,94$ & 9,9 & $6,23 *$ & $3,03-12,82$ & 3612 \\
\hline Total & 10,6 & - & - & 5,6 & - & - & 10186 \\
\hline \multicolumn{8}{|c|}{ Complexidade } \\
\hline Sem UTI & 7,6 & 1 & - & 4,7 & 1 & - & 4756 \\
\hline Com UTI & 13,3 & $1,75^{*}$ & $1,32-2,33$ & 6,3 & 1,36 & $0,89-2,08$ & 5430 \\
\hline Total & 10,6 & - & - & 5,6 & - & - & 10186 \\
\hline \multicolumn{8}{|c|}{ Residente no MRJ } \\
\hline Sim & 9,4 & 1 & - & 5,4 & 1 & - & 8973 \\
\hline Não & 20,7 & 2,21 * & $1,45-3,36$ & 7,0 & 1,30 & $0,63-2,71$ & 1160 \\
\hline Total & 10,7 & - & - & 5,6 & - & - & 10133 \\
\hline \multicolumn{8}{|c|}{ Peregrinação } \\
\hline Não & 7,9 & 1 & - & 5,1 & 1 & - & 7742 \\
\hline $\operatorname{Sim}$ & 19,0 & 2,41 * & $1,75-3,31$ & 7,2 & 1,41 & $0,83-2,38$ & 2422 \\
\hline Total & 10,5 & - & - & 5,6 & - & - & 10164 \\
\hline
\end{tabular}

$\mathrm{RR}=$ risco relativo; $\mathrm{MRJ}=$ município do Rio de Janeiro. ${ }^{*} \boldsymbol{p}<0,05$ 
Figura 1

Causa básica do óbito neonatal e pós-neonatal segundo o peso ao nascer e o tipo de prestador.

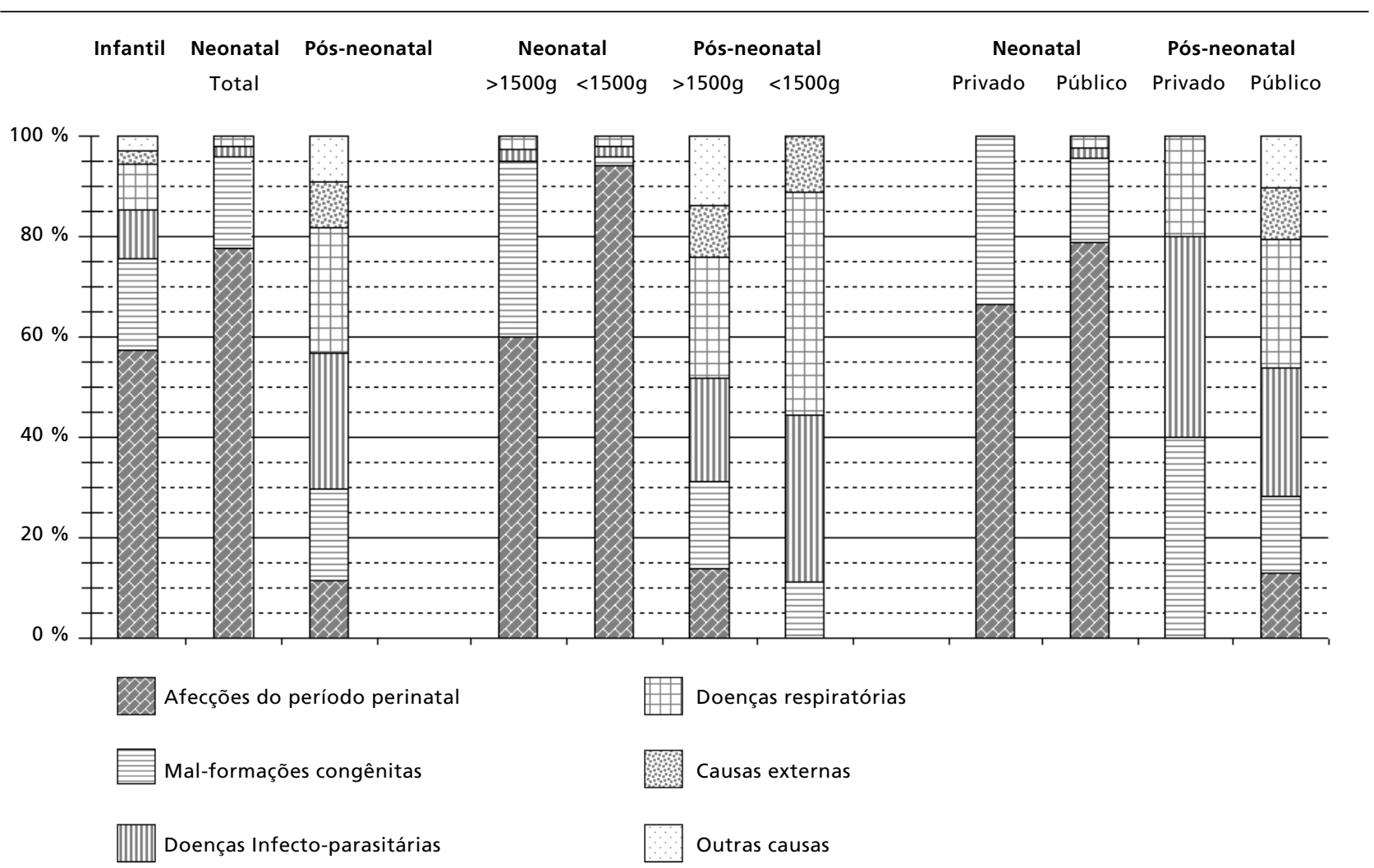

havendo uma contribuição relevante das causas externas $9,1 \%$, além das comumente encontradas nesse componente, como as doenças infecto-contagiosas e parasitárias (25\%) e as doenças respiratórias (25\%) (Figura 1).

Segundo o peso ao nascer, nos RN com peso $>1500 \mathrm{~g}$ as APP corresponderam a $60 \%$ dos óbitos neonatais e para aqueles com peso $<1500 \mathrm{~g}$ essa proporção foi de $94 \%$. Por outro lado, nos RN com $>1500 \mathrm{~g}$ foi maior o percentual de óbitos por malformação congênita (35\%) (Figura 1).

Segundo o tipo de prestador, a parcela de óbito neonatal por APP foi $21 \%$ maior nos hospitais do SUS que nos privados, $79 \%$ e $66 \%$ respectivamente (Figura 1). Cabe ressaltar que o percentual recai sobre a taxa, maior nos hospitais do SUS (estrato $2 \mathrm{e}$ 3) que nos privados, 14,0 e 2,8 por mil NV, respectivamente. Sendo assim, a taxa de mortalidade neonatal por APP foi de 11,0 óbitos por mil NV para os hospitais do SUS e de 1,8 por mil NV para os privados, ou seja, seis vezes maior no primeiro que no segundo grupo.

A contribuição das doenças infecto-parasitárias e doenças respiratórias para a mortalidade pós- neonatal foram bastante relevantes tanto nos hospitais privados $(40 \%$ e $20 \%)$ quanto nos hospitais públicos (23\% e $26 \%$, respectivamente). Nos hospitais públicos, entretanto, ainda foram encontradas proporções importantes de óbitos por APP (12\%) e por causas externas (12\%) enquanto que nos privados as mal-formações congênitas foram mais relevantes para esse componente (40\%). Apesar do maior percentual de óbitos pós-neonatais por doenças infecto-parasitárias nos hospitais privados que nos públicos (40\% e $23 \%$ ), quando se avalia esse resultado por meio da taxa ( $40 \%$ de 1,6 óbitos por mil NV e $23 \%$ de 7,5 por mil NV), observamos que a taxa de mortalidade pós-neonatal por essa causa foi três vezes menor nos hospitais privados $(0,6$ óbitos por mil NV) que nos públicos (1,7 por mil NV).

\section{Discussão}

Um dos grandes desafios dos estudos que utilizam amostras é a representatividade da população base. Nessa pesquisa a amostra foi calculada a partir dos nascimentos hospitalares ocorridos no município do 
Rio de Janeiro, independentemente do município de residência da mulher, ou seja, a opção foi a de avaliar as puérperas e $\mathrm{RN}$ assistidos no município e não apenas seus residentes.

Uma limitação do estudo se refere ao acompanhamento dos nascimentos até a ocorrência do óbito infantil. Como esse foi feito de forma pontual, por meio do "linkage" com o banco de dados do SIM, possíveis erros relacionados a esse sistema de informação, assim como ao processo de "linkage" não podem ser ignorados. Por outro lado, o SIM tem mostrado ampla cobertura no município do Rio de Janeiro $^{7}$, o que possibilitou a sua utilização neste estudo.

A semelhança das taxas de mortalidade neonatal e pós-neonatal na população do estudo com as do município do Rio de Janeiro reafirma a representatividade dessa amostra. A pequena diferença encontrada para o componente neonatal pode ser atribuída a períodos de tempo distintos, já que a abrangência da amostra foi de meados de 1999 a março de 2001, enquanto que no Rio de Janeiro a taxa considerada foi a do ano de 2000.

O fato de parte dos óbitos identificados no trabalho de campo não terem suas declarações de óbito encontradas no SIM surpreende, uma vez que todos esses óbitos ocorreram em maternidades localizadas no município do Rio de Janeiro, independentemente do local de residência da mãe. Esses óbitos podem não ter sido notificados, mas também não se descarta a possibilidade desse achado ser decorrente da ausência ou do mau preenchimento de dados do SIM, utilizados para o "linkage", ou por alguma falha nesse processo.

Óbitos neonatais notificados como óbitos fetais reflete que ainda é comum em nosso meio ignorar óbitos de crianças muito prematuras ou ocorridos logo após o nascimento, o que implica na notificação incorreta como óbito fetal pela ausência da emissão da declaração de Nascido Vivo (DN). Resultado semelhante foi encontrado em Santo André, SP por Almeida e Mello8 que apontaram uma possível concentração dessa falha em recém-nascidos (RN) com menor viabilidade.

Devido à maior disponibilidade de serviços de alta tecnologia para a atenção perinatal e, por conseqüência, o atendimento dos $\mathrm{RN}$ em estado mais grave nos hospitais municipais e federais, foram estimadas e eram esperadas para esses, maiores taxas de mortalidade neonatal e pós-neonatal que nos privados não conveniados ao SUS (Tabela 1). Além disso, segundo Leal et al., 4 esses estabelecimentos recebem maiores proporções de mães adolescentes, com pouca escolaridade e baixo número de consultas no pré-natal. Cabe ressaltar que apesar do alto risco da população atendida, taxas de cerca de 20 óbitos neonatais por mil NV e de 10 pós-neonatais por mil $\mathrm{NV}$ são consideradas extremamente altas e poderiam ser reduzidas em curto prazo por meio de intervenções diretas sobre a parcela de óbitos potencialmente evitáveis.

A maior taxa de mortalidade neonatal nas maternidades que dispunham de UTI neonatal do que naquelas sem esse recurso indica que as crianças mais graves foram referenciadas adequadamente e estão nascendo em locais com estrutura de maior complexidade. Resultados não apresentados revelaram que nessas maternidades nasceram duas vezes mais crianças com baixo peso ao nascer e/ou prematuras e $40 \%$ mais com algum defeito congênito.

Para as puérperas oriundas de outros municípios que não o Rio de Janeiro, a maior taxa de mortalidade neonatal pode ser atribuída ao fato de o município do Rio de Janeiro ser referência para atendimento de crianças de risco para todo o Estado, sendo maior a parcela de nascimentos com algum tipo de risco materno ou infantil entre as não moradoras no município.

No grupo das puérperas que peregrinaram (tentaram realizar o parto em outros estabelecimentos sem sucesso), a maior taxa de mortalidade neonatal sugere um possível efeito imediato no aumento do risco de óbito neonatal pela demora na assistência, como pode também ser devido à associação entre a peregrinação e a gravidez de alto risco, já que essas gestantes tendem a enfrentar maior dificuldade para a internação. Por outro lado, existe associação entre peregrinação e condições de vida desfavoráveis. Com essa mesma população amostral, foi demonstrado que nos hospitais vinculados ao SUS, 2 vezes mais mulheres não conseguiram ser atendidas no primeiro hospital em comparação com aquelas cujo parto foi realizado nas instituições privadas. ${ }^{4}$

$\mathrm{O}$ alto percentual de causas mal definidas poderia ser reduzido se o município adotasse um modelo semelhante ao do Instituto Materno Infantil de Pernambuco (IMIP), onde todas as Declarações de Óbitos passam por processo sistemático de revisão, tendo o prontuário médico como "Padrãoouro". 9 Dessa forma, reduz-se quase a zero a chance de óbito com causa básica mal definida, salvo em crianças que chegam sem vida ao serviço ou aquelas que vão a óbito antes de um possível diagnóstico, nas primeiras $24 \mathrm{~h}$ de internação. ${ }^{9}$

Considerando a amostra como um todo, a elevada proporção de óbitos causados por doenças infecto-parasitárias e respiratórias apontam falhas nos programas de atenção primária à saúde infantil, 
bem como refletem as grandes desigualdades sociais da nossa população. 10

Segundo o peso ao nascer, no período pósneonatal, maiores proporções de óbitos por doenças infecto-parasitárias e respiratórias naqueles com $<1500 \mathrm{~g}$ reforçam a maior fragilidade dessas crianças, mesmo após o primeiro mês de vida.

A ocorrência não rara de óbitos neonatais por APP em crianças com $>1500 \mathrm{~g}$ (taxa de 2,3 óbitos por mil NV), nos leva a refletir sobre a qualidade da assistência ao pré-natal, parto e ao recém-nascido. Lansky et al.,11 utilizando a classificação de Wigglesworth, verificaram em Belo Horizonte que $40 \%$ dos óbitos perinatais poderiam ter sido evitados com a organização da rede assistencial e a melhoria do atendimento clínico à gestante e ao RN. No Estado de São Paulo foi observado que, com a exclusão das anomalias congênitas, as APP representaram quase que a causa exclusiva de mortalidade neonatal.12

\section{Referências}

1. SUS (Sistema Unico de Saúde). Indicadores e Dados Básicos de Saúde. Estatísticas vitais: nascidos vivos e indicadores de mortalidade. Taxas calculadas. Disponível em http://www.datasus.gov.br [2006 Dez 10].

2. Machado CJ. A literature review of record linkage procedures focusing on infant health outcomes. Cad Saúde Pública. 2004; 20: 362-71.

3. Theme Filha MM, Gama SGN, Cunha CB, Leal MC Confiabilidade do Sistema de Informações sobre Nascidos Vivos Hospitalares no Município do Rio de Janeiro, 19992001. Cad Saúde Pública 2004; 20 [Sup 1]: S83-91.

4. Leal MC, Gama SGN, Campos MR, Cavalini LT, Garbayo LS, Brasil CLP, Szwarcwald CL. Fatores associados a morbi-mortalidade perinatal em uma amostra de maternidades públicas e privadas do Município do Rio de Janeiro, 1999-2001.Cad Saúde Pública. 2004; 20 [Supl 1]: S20-33.

5. Newcombe HB. Record linkage: Methods for health and statistical studies, administration and busines. New York: Oxford University Press; 1989.

6. Camargo JR KR, Coeli CM. RECLINK: Aplicativo para o relacionamento de banco de dados implementando o método probabilistic record linkage. Cad Saúde Pública. 2000; 16: 439-47.

7. Szwarcwald CL, Leal MC, Andrade CLT, Borges PRSJ. Estimação da mortalidade infantil no Brasil: o que dizem as informações sobre óbitos e nascimentos do Ministério da Saúde. Cad Saúde Pública. 2002; 18: 1725-36.
Considerando o fato de a taxa de mortalidade neonatal ter sido sete vezes maior nos hospitais do SUS que nos hospitais privados, o maior percentual de óbitos neonatais por APP nesses estabelecimentos reafirma a extrema heterogeneidade entre os estratos.

A persistência de APP como causa de morte pósneonatal nos hospitais do SUS (taxa de 1,7 por mil $\mathrm{NV}$ ), apontam para uma deficiência na assistência especializada nesses estabelecimentos que estão conseguindo prolongar a vida do recém-nato, mas não evitar sua morte. Surpreende que na mesma proporção (taxa de 1,7 por mil NV) ocorreram óbitos por causas externas nesses estratos, levando-nos a questionar o ambiente em que estaria inserida essa criança. No município de São Paulo, Machado e Hill,13 também encontraram relevante proporção de causas externas na mortalidade de menores de um ano.

8. Almeida MF, Mello JMHPM. The use of the "linkage" technique of information systems in cohort studies on neonatal mortality. Rev Saúde Pública. 1996; 30: 141-7.

9. Vidal AS, Frias PG, Barreto FMP, Vanderley LGM, Felisberto E. Óbitos infantis evitáveis em hospital de referência estadual do Nordeste Brasileiro. Rev Bras Saúde Matern Infant. 2003; $3: 281-9$.

10. França E, Souza JM, Guimarães MDC, Goulart EMA, Colosimo E, Antunes CMF. Associação entre fatores socioeconômicos e mortalidade infantil por diarréia, pneumonia e desnutrição em região metropolitana do Sudeste do Brasil: um estudo caso-controle. Cad Saúde Pública. 2001; 17: 1437-47.

11. Lansky S, França E, Leal MC. Mortes perinatais evitáveis em Belo Horizonte, Minas Gerais, Brasil, 1999. Cad Saúde Pública. 2002; 18: 1389-400.

12. Kilsztajn S, Rossbach A, Carmo MSN, Sugahara GTL. Assistência pré-natal, baixo peso e prematuridade no Estado de São Paulo, 2000. Rev Saúde Pública. 2003; 37: 303-10.

13. Machado CJ, Machado CJ, Hill K. Determinantes da mortalidade neonatal e pós-neonatal no Município de São Paulo. Rev Bras Epidemiol. 2003, 6: 345-58.

Recebido em 20 de dezembro de 2006

Versão final apresentada em 20 de janeiro de 2007

Aprovado em 6 de fevereiro de 2007 